\section{Structural adaptations of photosynthetic complex I enable ferredoxin-dependent electron transfer}

\author{
Jan M. Schuller ${ }^{1 *}$, James A. Birrell ${ }^{2}$, Hideaki Tanaka ${ }^{3,4}$, Tsuyoshi Konuma ${ }^{5}$, \\ Hannes Wulfhorst ${ }^{6,7}+$, Nicholas Cox $^{2,8}$, Sandra K. Schuller ${ }^{9}$, Jacqueline Thiemann ${ }^{6}$, \\ Wolfgang Lubitz ${ }^{2}$, Pierre Sétif ${ }^{\text {To }}$, Takahisa Ikegami ${ }^{5}$, Benjamin D. Engel ${ }^{11}$, \\ Genji Kurisu $^{3,4 *}$, Marc M. Nowaczyk ${ }^{6 *}$
}

\begin{abstract}
Photosynthetic complex I enables cyclic electron flow around photosystem I, a regulatory mechanism for photosynthetic energy conversion. We report a 3.3-angstrom-resolution cryo-electron microscopy structure of photosynthetic complex I from the cyanobacterium Thermosynechococcus elongatus. The model reveals structural adaptations that facilitate binding and electron transfer from the photosynthetic electron carrier ferredoxin. By mimicking cyclic electron flow with isolated components in vitro, we demonstrate that ferredoxin directly mediates electron transfer between photosystem I and complex I, instead of using intermediates such as NADPH (the reduced form of nicotinamide adenine dinucleotide phosphate). A large rate constant for association of ferredoxin to complex I indicates efficient recognition, with the protein subunit NdhS being the key component in this process.
\end{abstract}

T wo light-driven electron transport pathways operate in all organisms that perform oxygenic photosynthesis: linear and cyclic electron flow. In linear electron flow (LEF), two photochemical reaction centers (photosystems I and II) act in series to drive the synthesis of adenosine triphosphate (ATP) and the reduced form of nicotinamide adenine dinucleotide phosphate (NADPH), whereas cyclic electron flow (CEF), powered by only photosystem I (PSI), leads solely to the formation of ATP. The contribution of each pathway varies in response to the environment (e.g., light quality), and organisms in which CEF is inactivated are functionally impaired (1).

Photosynthetic complex I of plant chloroplasts and cyanobacteria $(2,3)$ has been implicated in

\footnotetext{
Department of Structural Cell Biology, Max Planck Institute of Biochemistry, 82152 Martinsried, Germany. ${ }^{2}$ Max Planck Institute for Chemical Energy Conversion, 45470 Mülheim an der Ruhr, Germany. ${ }^{3}$ Institute for Protein Research, Osaka University, Suita, Osaka 565-0871, Japan. ${ }^{4}$ Department of Macromolecular Science, Graduate School of Science, Osaka University, Toyonaka 560-0043, Japan. ${ }^{5}$ Graduate School of Medical Life Science, Yokohama City University, 1-7-29 Suehiro-cho, Tsurumi-ku, Yokohama 230-0045, Japan. ${ }^{6}$ Plant Biochemistry, Faculty of Biology and Biotechnology, Ruhr University Bochum, 44780 Bochum, Germany. ${ }^{7}$ Daiichi Sankyo Deutschland GmbH, Zielstattstr. 48, 81379 München, Germany. ${ }^{8}$ Research School of Chemistry, Australian National University, Canberra, ACT 2601, Australia. ${ }^{9}$ Gene Center and Department of Biochemistry, Ludwig-Maximilians-Universität München, Feodor-Lynen-Str. 25, 81377 Munich, Germany.

${ }^{10}$ Institut de Biologie Intégrative de la Cellule (I2BC), IBITECS, CEA, CNRS, Université Paris-Saclay, F-91198 Gif-sur-Yvette, France. ${ }^{11}$ Department of Molecular Structural Biology, Max Planck Institute of Biochemistry, 82152 Martinsried, Germany.

*Corresponding author. Email: janschu@biochem.mpg.de (J.M.S.); gkurisu@protein.osaka-u.ac.jp (G.K.); marc.m. nowaczyk@rub.de (M.M.N.) †Present address: Daiichi Sankyo
} Deutschland GmbH, Zielstattstr. 48, 81379 Munich, Germany.
$\mathrm{CEF}$, taking electrons from and indirectly reinjecting them into PSI. It is structurally and functionally related to respiratory complex I from mitochondria and bacteria (4-6), but it lacks the peripheral dehydrogenase module (N-module), comprising subunits NuoE, NuoF, and NuoG (see table S1 for nomenclature in different organisms). This module catalyzes NADH (the reduced form of nicotinamide adenine dinucleotide) oxidation and contains five out of eight iron-sulfur (Fe-S) clusters. Biochemical and proteomic analyses of photosynthetic complexes have discovered at least eight distinct subunits required to assemble fully functional photosynthetic complex I (7-13). There is evidence that ferredoxin (Fd) likely mediates electron transfer between photosynthetic complex I and PSI (14), probably within a large supercomplex (15), but this process has not been directly observed. Furthermore, the structural adaptions that enable the photosynthetic complex to perform its distinct role remain uncharacterized.

We purified photosynthetic complex I from the thermophilic cyanobacterium Thermosynechococcus elongatus (fig. S1 and tables S2 and S3) and determined the structure by cryo-electron microscopy (cryo-EM) single-particle analysis to an overall resolution of $3.3 \AA$ (Fig. 1; figs. S2 and S3; and table S4). We constructed models from homologous subunits or de novo where no model was available (fig. S4), except for NdhV, which binds transiently (7) and was not observed.

Photosynthetic complex I transfers electrons to the terminal acceptor plastoquinone. The quinonebinding site is coupled to the proton-pumping machinery in the membrane by a highly conserved charge-redistribution cascade (figs. S5 and $\mathrm{S} 6$ ), but the exact coupling mechanism remains elusive (16-19).

The photosynthesis-specific single-spanning membrane proteins NdhQ and NdhP (8) bind to either side of the NdhD protein, with $\mathrm{NdhQ}$ fixing the very long horizontal helix of $\mathrm{NdhF}$ (fig. S7). NdhP forms a small hydrophobic cavity to which a molecule of $\beta$-carotene is bound. A lipid molecule (monogalactosyldiacylglycerol) binds between the NdhD and NdhF proton channels and appears to be stabilized by the $\beta$-carotene molecule. Both molecules probably serve as "molecular glue," similar to lipids found in complex I of other species $(18,20)$, to help assemble the proton-pumping membrane arm of the complex and to stabilize the NdhF binding interface. A third photosynthesis-specific membrane subunit, $\mathrm{NdhL}$, binds to the N terminus of NdhA to form an extended heel under the peripheral arm (fig. S5).

The N-module subunits responsible for NADH oxidation are not present in photosynthetic complex I. Instead, the peripheral arm of the cyanobacterial complex (Q-module) contains photosynthesis-specific subunits (NdhM, NdhN, NdhO, and NdhS) (figs. S8 and S9), which bind to the conserved, nonmembrane subunits of complex I (NdhH, NdhI, NdhJ, and NdhK). The latter four subunits harbor three [4Fe-4S] clusters in addition to the quinone-binding site and have elongated termini that are conserved within the green linage (figs. S10 to S12). NdhM and NdhN are located at one side of the peripheral arm and form multiple interactions with the conserved [4Fe-4S]-carrying complex I subunits by binding to their elongated termini and covering otherwise solvent-exposed hydrophobic patches. NdhO has a globular fold and packs tightly to the side of NdhJ via a hydrophobic binding interface, covering the same space that is occupied by the speciesspecific protein TTHA1528 in T. thermophilus respiratory complex I (21). Furthermore, the photosynthesis-specific NdhS subunit, previously implicated in Fd binding (22), is located in a Vshaped groove formed by the NdhI protein, at a similar location to subunit Nqo15 within the $\mathrm{N}$-module.

Our cryo-EM structure resolves the positions and conserved coordination of three [4Fe-4S] clusters, corresponding to the previously identified clusters N6a, N6b, and N2 in respiratory complex I (Fig. 2A). Electron paramagnetic resonance (EPR) measurements on chemically reduced samples quantitatively identify all three clusters (components 1, 2, and 3 in Fig. 2B). Components 1 and 2 are similar to the respiratory complex I signals of $\mathrm{N} 2$ and N6b $(23,24)$, whereas component 3 bears little resemblance to N6a, in terms of width and structure (tables S6 and S13). The difference is due to either the proximity of N6a to N6b or the fact that N6a is surface exposed in photosynthetic complex I, as opposed to being buried in the protein, allowing it to act as the site of electron injection (see supplementary text).

To test the hypothesis that reduced Fd can inject electrons into photosynthetic complex I to enable CEF, we used absorption kinetics in 


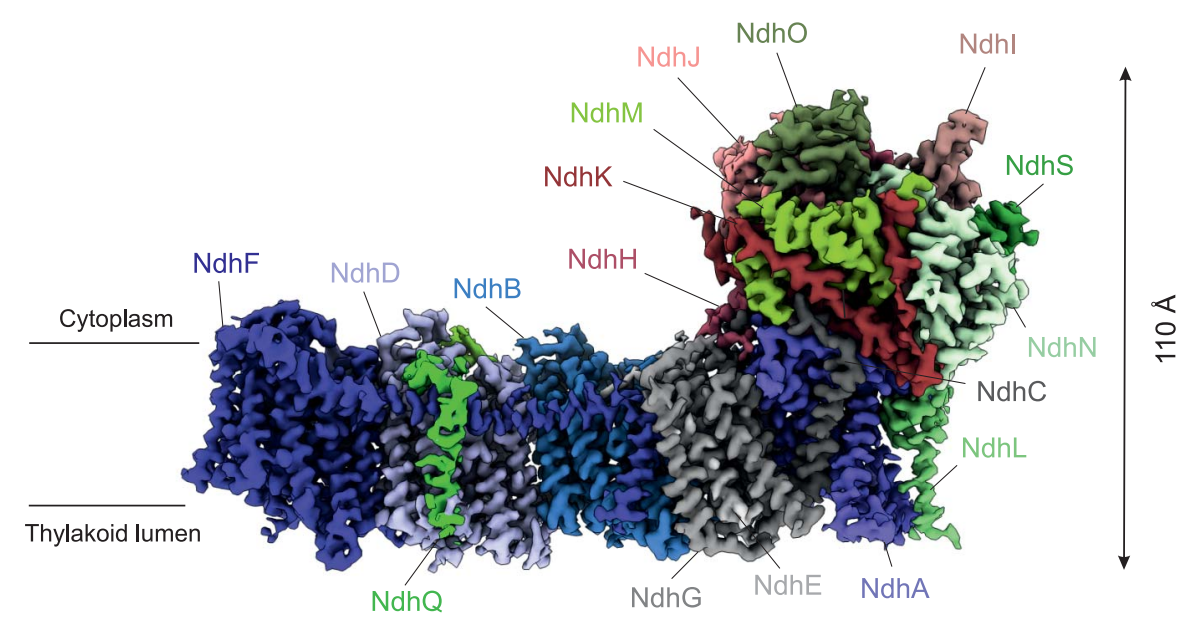

$190 \AA$

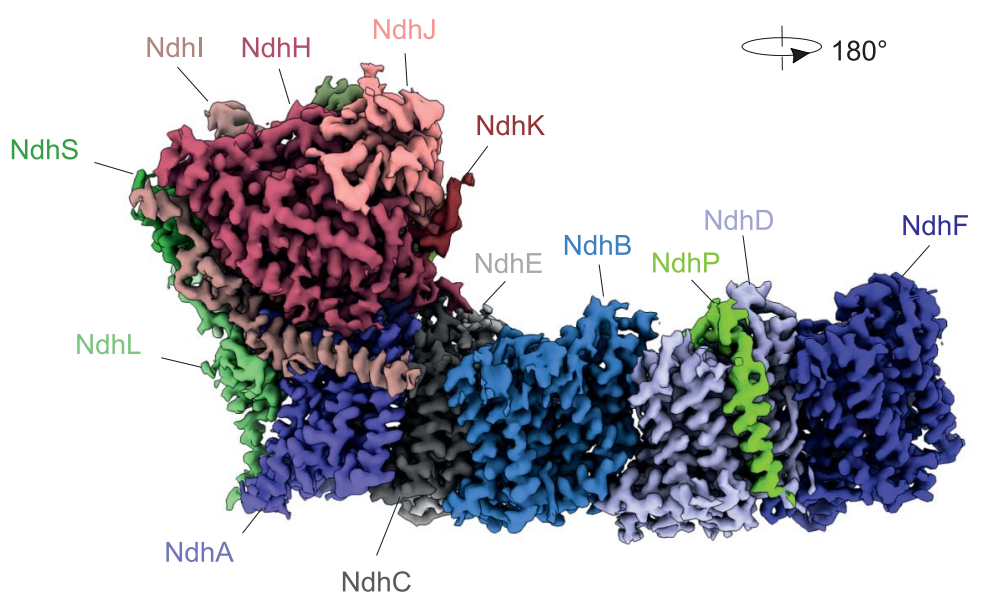

Fig. 1. Cryo-EM map of photosynthetic complex I from T. elongatus segmented by subunit. Eighteen subunits are colored and named accordingly (photosynthesis-specific subunits NdhL, NdhM, NdhN, NdhO, NdhP, NdhQ, and NdhS; other nonmembrane subunits $\mathrm{NdhH}, \mathrm{Ndhl}$, NdhJ, and NdhK; and other membrane subunits NdhA, NdhB, NdhC, NdhD, NdhE, $\mathrm{NdhF}$, and $\mathrm{NdhG}$ ).

the submillisecond time range. Electron transfer reactions were monitored in vitro with samples containing purified PSI, Fd, and photosynthetic complex I (Fig. 2, C and D). Single PSI turnover was triggered with a short laser flash (Fig. 2C), and subsequent electron transfer was monitored by ultraviolet-visible light absorption (see supplementary text). Submicrosecond PSI charge separation and stabilization (fig. S14) was followed by Fd reduction by the terminal [4Fe-4S] cluster of PSI, dissociation of reduced $\mathrm{Fd}\left(\mathrm{Fd}_{\mathrm{red}}\right)$ from PSI, and reduction of a [4Fe-4S] cluster in photosynthetic complex I by $\mathrm{Fd}_{\text {red }}$ (Fig. 2C). The kinetics of the latter reaction were fitted to a biexponential function with rates of 245 and $1280 \mathrm{~s}^{-1}$ (Fig. 2C). With the conservative assumption that the slowest component $\left(245 \mathrm{~s}^{-1}\right)$ corresponds to the association of $\mathrm{Fd}_{\text {red }}$ to the photosynthetic complex I, we calculated a lower limit of $k_{2 \text { FdComplexI }}=1.0 \times 10^{9} \mathrm{M}^{-1} \mathrm{~s}^{-1}$ for binding. This association constant is clearly larger than values previously measured for other $\mathrm{Fd}$ partner proteins $(25-27)$. We conclude that $\mathrm{Fd}$ participates in CEF via photosynthetic complex I and that Fd recruitment to complex I is very efficient.

The above analyses converge upon the hypothesis that the surface of the peripheral arm near the N6a cluster of photosynthetic complex I is responsible for Fd binding (Fig. 3A). We identified a putative $\mathrm{Fd}$ binding site in this region, guided by the surface charge at a tripartite interface formed by NdhK, NdhI, and NdhS (Fig. $3 \mathrm{~B}$ ). This surface area faces toward the missing [4Fe-4S] cluster N5 of the N-module of respiratory complex I (Fig. 2D).

Recent functional studies suggested that the photosynthesis-specific subunit NdhS plays an important role in CEF of Arabidopsis thaliana $(22,28)$ and Synechocystis sp. PCC 6803 (13). However, because NdhS has no prosthetic group such as an Fe-S cluster or flavin, it is still elusive how it is involved in electron transfer of photosynthetic complex I. We confirmed that unbound $\mathrm{NdhS}$ adopts the same overall structure as in the full complex I by solving the x-ray crystal structure of recombinant $\mathrm{NdhS}$ at $1.90-\AA$ resolution (table S5 and figs. S15 and S16). To assess the sites and mode of interaction between Fd and NdhS, we performed nuclear magnetic resonance (NMR) chemical shift perturbation experiments using ${ }^{15} \mathrm{~N}$-labeled $\mathrm{Fd}$ or $\mathrm{NdhS}$ with the nonlabeled counterpart, and vice versa (Fig. 3D and figs. S16 to S18). The NMR chemical shift perturbation indicated that the interaction site on $\mathrm{NdhS}$ was primarily located in its C-terminal region, from $\mathrm{Glu}^{104}$ (E104) to the $\mathrm{C}$ terminus, a domain that was not resolved in either the x-ray or cryo-EM structure owing to its high flexibility (Fig. 3, $\mathrm{C}$ and D). A similar "fly-casting" mechanism leads to fast electron transfer between $\mathrm{Fd}$ and ferredoxin:NADP ${ }^{+}$reductase $(25,29)$, and it might be also responsible for the fast association between Fd and photosynthetic complex I. The C-terminal segment of NdhS contains five positively charged Lys residues, which likely "catch" the negatively charged patch of Fd through an electrostatic interaction. We propose that NdhS serves as a foothold for Fd binding by tuning the binding angle of Fd toward NdhI, the catalytic subunit with a redox center next to NdhS (Fig. 3C and fig. S19), as is the case for the variable subunit of ferredoxin:thioredoxin reductase and for the PsaE subunit of PSI.

Our structure of photosynthetic complex I suggests that adaptation of modular domains and interfaces contributes to functional differences between it and homologous complexes. The minimal functional unit of the Q-module for Fd-dependent electron transfer is shared with membrane-bound hydrogenase from Archaea (30), thus suggesting that they also share the minimal required interaction site for Fd binding. In respiratory complex I, the N-module is attached to the Q-module to enable NADH oxidation (21), whereas in photosynthetic complex I, extensions and accessory subunits (including NdhS) facilitate highly efficient electron transfer from Fd. The photosynthesis-specific structural elements may also mediate supercomplex formation with PSI, which is proposed to further optimize CEF in cyanobacteria and plants (15).

\section{REFERENCES AND NOTES}

1. Y. Munekage et al., Nature 429, 579-582 (2004).

2. G. Peltier, E.-M. Aro, T. Shikanai, Annu. Rev. Plant Biol. 67, 55-80 (2016)

3. N. Battchikova, M. Eisenhut, E.-M. Aro, Cyanobacterial NDH-1 complexes: Novel insights and remaining puzzles Biochim. Biophys. Acta 1807, 935-944 (2011).

4. L. A. Sazanov, Nat. Rev. Mol. Cell Biol. 16, 375-388 (2015).

5. J. Hirst, M. M. Roessler, Energy conversion, redox catalysis and generation of reactive oxygen species by respiratory complex I. Biochim. Biophys. Acta 1857 , 872-883 (2016)

6. C. Wirth, U. Brandt, C. Hunte, V. Zickermann, Structure and function of mitochondrial complex I. Biochim. Biophys. Acta 1857, 902-914 (2016). 
Fig. 2. Assignment of Fe-S clusters by EPR spectroscopy and in vitro electron transfer kinetics from PSI via Fd toward complex I. (A) [4Fe-4S] clusters N6a, N6b, and N2 with coordinating subunits $\mathrm{Ndhl}$ (dark salmon) and NdhK (ruby) compared with the corresponding $T$. thermophilus subunits Nqo9 and Nqo6 [gray, Protein Data Bank (PDB) 4 HEA (21)]. (B) An EPR spectral simulation (red dashed line) of the spectrum at $10 \mathrm{~K}$ (black line), along with simulated spectra for the individual components (green, blue, and pink lines) and their associated $g$-values. (C) Changes in flash-induced absorption at $580 \mathrm{~nm}\left(\Delta \mathrm{A}_{580} \mathrm{~nm}\right)$, attributed to $\mathrm{Fd}$ reduction by PSI (left) and complex I reduction by $\mathrm{Fd}_{\text {red }}$ (right). These changes correspond to differences between signals 1,2 , and 3 , recorded in three different cuvettes containing PSI, PSI-Fd, and PSI-Fd-complex I, respectively (see individual measurements in fig. S14). The left graph shows the difference between signals 2 and 1 . The slow component after 0.2 ms was fitted by a single rising exponential function (black curve; rate, $1379 \mathrm{~s}^{-1}$ ). The difference is due to oxidized $\mathrm{Fd}\left(\mathrm{Fd}_{\mathrm{ox}}\right)$ binding to PSI (which follows $\mathrm{Fd}_{\text {red }}$ dissociation) with a second-order rate constant of $2.8 \times 10^{8} \mathrm{M}^{-1} \mathrm{~s}^{-1}$ (see materials and methods). The downward arrow corresponds to the reduction of Fd by the PSI terminal acceptor. The right graph shows the difference between signals 3 and 2. To account for the lag preceding the signal rise due to complex I reduction by $\mathrm{Fd}_{\text {red }}$, the kinetics were fitted by a biexponential function with rates of 245 and $1280 \mathrm{~s}^{-1}$ (black curve). The upward arrow corresponds to the reduction of a [4Fe-4S] cluster in photosynthetic complex I by Fd. The PSI, Fd, and complex I concentrations were $0.08,5.0$, and $0.24 \mu \mathrm{M}$, respectively. (D) Superposition of photosynthetic complex I (green) with the T. thermophilus complex I [gray, PDB 4HEA (21)]. The N-module-which contains the NADH binding site, the flavin mononucleotide (FMN) cofactor, and the six Fe-S clusters Nla, N1b, N3, N4, N5, and N7 that transfer electrons from $\mathrm{NADH}$ (light blue arrows)-is missing from photosynthetic complex I. In vitro, electrons are transferred after light-induced charge separation from PSI [PDB 5ZF0 (31)] via Fd [PDB 5AUI (32)] toward the Q-module of photosynthetic complex I, as indicated by the red and dark blue arrows. The lightning bolt indicates application of a laser flash to excite PSI. PQ, plastoquinone.
A

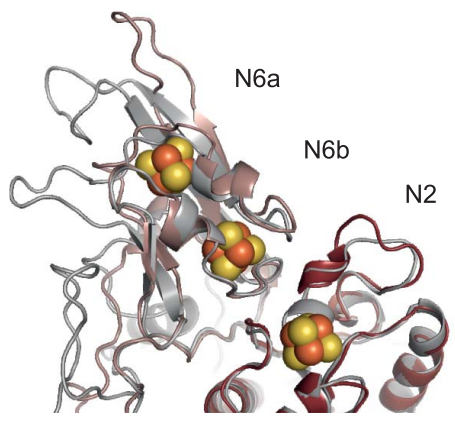

C
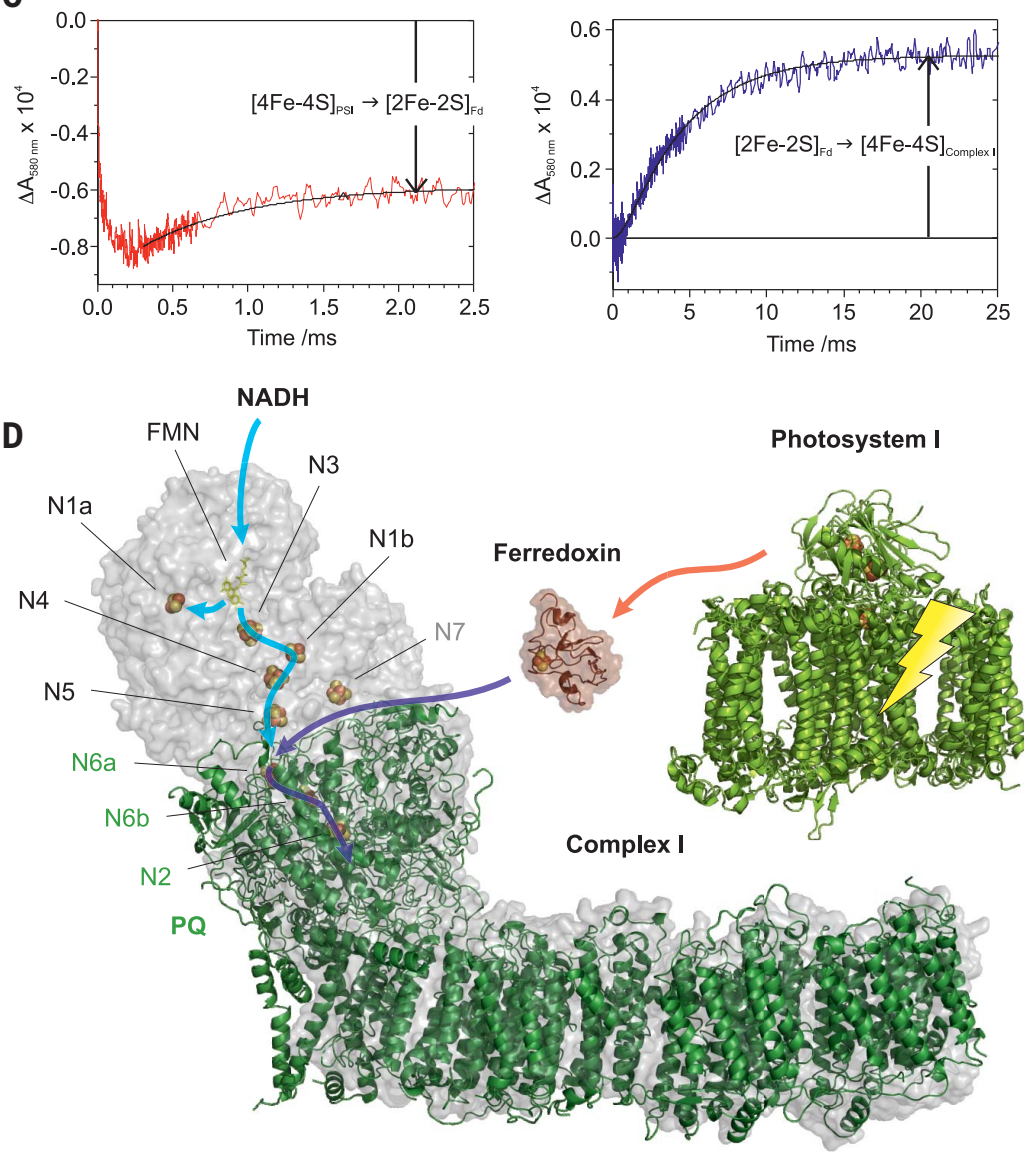

B

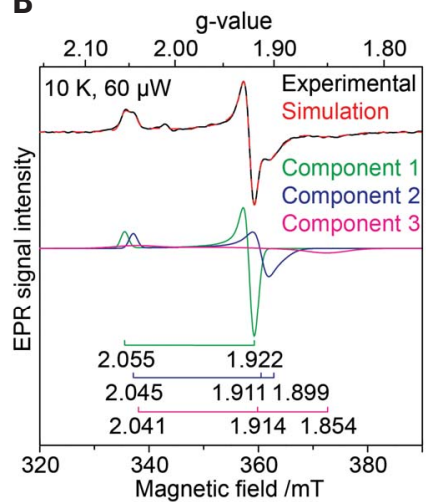

Fig. 3. Adaptation of the Q-module to Fd binding and the role of the NdhS $\mathbf{C}$ terminus. (A) Top view of the complex I surface with colored subunits. The potential Fd binding area is indicated by the yellow dashed circle. (B) Electrostatic potential surfaces on complex I (red for negative, white for neutral, and blue for positive) were calculated with the Adaptive Poisson-Boltzmann Solver (APBS) plug-in in PyMOL. (C) The C-terminal segment of $\mathrm{NdhS}$ (green ribbon structure) that was not resolved in the $\mathrm{x}$-ray and cryo-EM structures is drawn as a dotted line in an arbitrary position. K, Lys. (D) Weighted ${ }^{1} \mathrm{H} /{ }^{15} \mathrm{~N}$ chemical shift perturbations observed in $\left[{ }^{15} \mathrm{~N}\right]-\mathrm{NdhS}$ upon binding to nonlabeled Fd. ppm, parts per million.
A

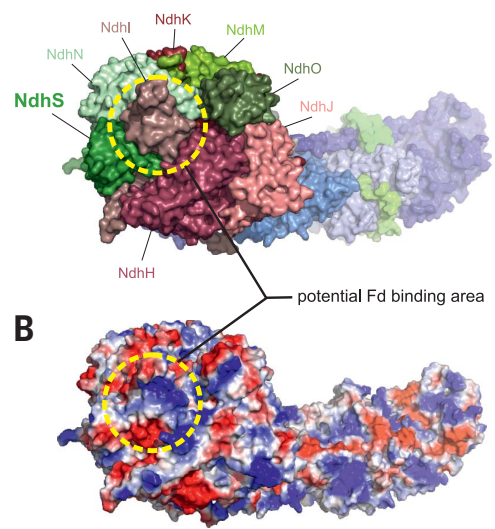

C

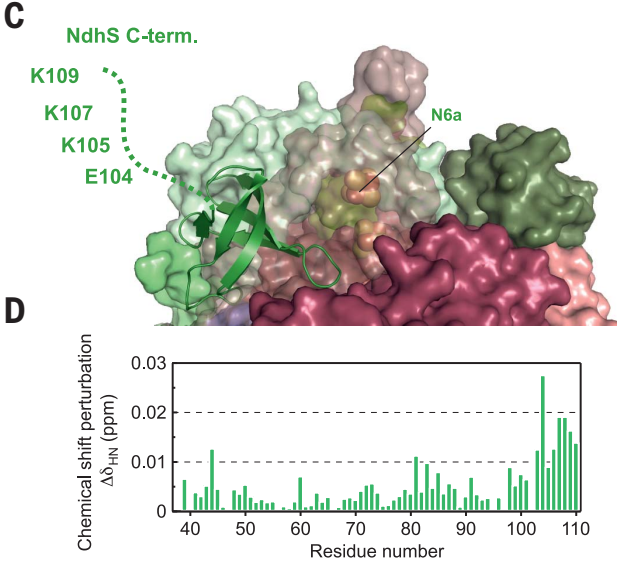


7. X. Fan, J. Zhang, W. Li, L. Peng, Plant J. 82, 221-231 (2015).

8. M. M. Nowaczyk et al., Biochemistry 50, 1121-1124 (2011).

9. T. Ogawa, Plant Physiol. 99, 1604-1608 (1992).

10. N. Battchikova, P. Zhang, S. Rudd, T. Ogawa, E.-M. Aro, J. Biol. Chem. 280, 2587-2595 (2005).

11. P. Prommeenate, A. M. Lennon, C. Markert, M. Hippler P. J. Nixon, J. Biol. Chem. 279, 28165-28173 (2004)

12. D. Rumeau et al., Plant Cell 17, 219-232 (2005).

13. N. Battchikova et al., J. Biol. Chem. 286, 36992-37001 (2011).

14. T. Friedrich, K. Steinmüller, H. Weiss, FEBS Lett. 367, 107-111 (1995).

15. L. Peng, H. Shimizu, T. Shikanai, J. Biol. Chem. 283 34873-34879 (2008).

16. V. R. I. Kaila, J. R. Soc. Interface 15, 20170916 (2018).

17. V. Zickermann et al., Science 347, 44-49 (2015).

18. A. A. Agip et al., Nat. Struct. Mol. Biol. 25, 548-556 (2018).

19. L. A. Sazanov, J. Bioenerg. Biomembr. 46, 247-253 (2014).

20. K. Fiedorczuk et al., Nature 538, 406-410 (2016).

21. R. Baradaran, J. M. Berrisford, G. S. Minhas, L. A. Sazanov, Nature 494, 443-448 (2013).

22. H. Yamamoto, L. Peng, Y. Fukao, T. Shikanai, Plant Cell 23, 1480-1493 (2011).

23. M. Narayanan et al., J. Biol. Chem. 288, 14310-14319 (2013).

24. T. Yano, S. Magnitsky, V. D. Sled', T. Ohnishi, T. Yagi, J. Biol. Chem. 274, 28598-28605 (1999)

25. N. Cassan, B. Lagoutte, P. Sétif, J. Biol. Chem. 280 25960-25972 (2005).

26. P. Sétif et al., Biochemistry 48, 2828-2838 (2009).
27. A. P. Srivastava, D. B. Knaff, P. Sétif, Biochemistry 53 , 5092-5101 (2014).

28. H. Yamamoto, T. Shikanai, J. Biol. Chem. 288, 36328-36337 (2013).

29. M. Maeda et al., Biochemistry 44, 10644-10653 (2005)

30. H. Yu et al., Cell 173, 1636-1649.e16 (2018).

31. H. Kubota-Kawai et al., Nat. Plants 4, 218-224 (2018).

32. R. Mutoh et al., Biochemistry 54, 6052-6061 (2015).

\section{ACKNOWLEDGMENTS}

We thank F. Hara, K. Umeno, N. Hamaoka, Y. Misumi, J. Y. Kim, C. König, M. Völkel, and R. Oworah-Nkruma for excellent technical assistance and Y. Miyanoiri, E. Yamashita, and A. Nakagawa for support during data collection by x-ray and NMR analysis. J.M.S. is gratefu to $E$. Conti for scientific independence and great mentorship and to J. M. Plitzko and W. Baumeister for access to the cryo-EM infrastructure and early career support. P.S. thanks A. Boussac and B. Lagoutte for purification of PSI and Fd. M.M.N. is grateful to his mentor M. Rögner for generous support. Synchrotron radiation experiments were performed at BL44XU of SPring-8, Japan (proposa no. 2017B6500). Funding: Financial support was provided by the Max Planck Society, the Cluster of Excellence RESOLV (EXC 1069 to W.L., N.C. and M.M.N.) funded by the German Research Council (DFG), the DFG research unit FOR2092 (EN 1194/1-1 to B.D.E. and NO 836/3-2 to M.M.N.), the DFG priority program 2002 (NO 836/4-1 to M.M.N.), DFG grant N0 836/1-1 (to M.M.N.), the Australian Research Council (FT140100834 to N.C.), JST-CREST (JPMJCR13M4 to G.K.), MEXT-KAKENHI (16H06560 to G.K.), the French Infrastructure for Integrated Structural Biology/FRISB ANR-10-INSB-05 (P.S.), and the International Joint Research Promotion Program, Osaka University (G.K. and M.M.N.). Author contributions: J.M.S. J.A.B., N.C. W.L., P.S., B.D.E, G.K and
M.M.N. conceived the research, prepared the figures, and wrote the manuscript with the contributions of all other authors.

M.M.N. coordinated the activities. Preparation of complex I, biochemical analysis, and mass spectrometry were done by H.W., J.T., and M.M.N. J.M.S. and B.D.E. performed the cryo-EM analysis and built the structural model with the help of S.K.S. and G.K. Flash-absorption spectroscopy was carried out by P.S. EPR experiments were conducted by J.A.B. and N.C. and NMR and x-ray analysis by H.T., T.K., T.I., and G.K All authors approved the final version of the manuscript. Competing interests: The authors declare no competing interests. Data and materials availability: The cryo-EM density map is deposited in the Electron Microscopy Data Bank under accession number EMD-0281. The atomic models of the cryo-EM and $x$-ray structures are deposited in the worldwide Protein Data Bank under accession numbers $6 \mathrm{HUM}$ and $6 \mathrm{~A} 7 \mathrm{~K}$, respectively. All other data are available in the manuscript or the supplementary materials.

\section{SUPPLEMENTARY MATERIALS}

www.sciencemag.org/content/363/6424/257/suppl/DC1 Materials and Methods

Supplementary Text

Figs. S1 to S19

Tables S1 to S6

References (33-88)

20 September 2018; accepted 6 December 2018 Published online 20 December 2018

10.1126/science.aau3613 


\section{Science}

\section{Structural adaptations of photosynthetic complex I enable ferredoxin-dependent electron transfer}

Jan M. Schuller, James A. Birrell, Hideaki Tanaka, Tsuyoshi Konuma, Hannes Wulfhorst, Nicholas Cox, Sandra K. Schuller, Jacqueline Thiemann, Wolfgang Lubitz, Pierre Sétif, Takahisa Ikegami, Benjamin D. Engel, Genji Kurisu and Marc M. Nowaczyk

Science 363 (6424), 257-260.

DOI: 10.1126/science.aau3613originally published online December 20, 2018

\section{Plugging into the pump}

Photosynthetic organisms use light to fix carbon dioxide in a process that requires both chemical reducing equivalents and adenosine triphosphate (ATP). Balancing the ratio of these inputs is accomplished by a short circuit in electron flow through photosynthetic complex I, a proton pump that contributes to ATP production but does not increase net reducing equivalents in the cell. Schuller et al. solved a cryo-electron microscopy structure of photosynthetic complex I (see the Perspective by Brandt) and went on to reconstitute electron transfer using the electron carrier protein ferredoxin.

Science, this issue p. 257; see also p. 230

ARTICLE TOOLS

SUPPLEMENTARY MATERIALS

RELATED CONTENT

REFERENCES

PERMISSIONS http://science.sciencemag.org/content/363/6424/257

http://science.sciencemag.org/content/suppl/2018/12/19/science.aau3613.DC1

http://science.sciencemag.org/content/sci/363/6424/230.full

This article cites 87 articles, 21 of which you can access for free http://science.sciencemag.org/content/363/6424/257\#BIBL

http://www.sciencemag.org/help/reprints-and-permissions

Use of this article is subject to the Terms of Service

Science (print ISSN 0036-8075; online ISSN 1095-9203) is published by the American Association for the Advancement of Science, 1200 New York Avenue NW, Washington, DC 20005. 2017 (C) The Authors, some rights reserved; exclusive licensee American Association for the Advancement of Science. No claim to original U.S. Government Works. The title Science is a registered trademark of AAAS. 